\title{
Reply to comment by N. Suciu and P. Knabner on "Spatial moments analysis of kinetically sorbing solutes in aquifer with bimodal permeability distribution"
}

\author{
M. Massabó, ${ }^{1}$ A. Bellin, ${ }^{2}$ and A. J. Valocchi ${ }^{3}$ \\ Received 10 February 2009; accepted 13 March 2009; published 9 May 2009.
}

Citation: Massabó, M., A. Bellin, and A. J. Valocchi (2009), Reply to comment by N. Suciu and P. Knabner on “'Spatial moments analysis of kinetically sorbing solutes in aquifer with bimodal permeability distribution," Water Resour. Res., 45, W05602, doi:10.1029/2009WR007815.

[1] We thank Suciu and Knabner [2009] for their commentary which discusses the influence of initial plume's shape on ergodicity of the second spatial moments. The discussion is based on previous findings of the authors [Suciu et al., 2008] showing that ergodicity of the second plume moments is a rather rare situation. In general, this discussion is relevant because ergodicity is typically invoked in order to approximate the spatial second moments of the actual plume $s_{l l}, l=1, \ldots, m$, where $m$ is the space dimensionality, with the variance of the particle's displacement $X_{l l}$. Conditions leading to ergodic second moments were investigated previously by Fiori and Janković [2005], while results on the evolution of the ensemble average of $s_{l l}$ have been presented earlier by other authors [see, e.g., Kitanidis, 1988; Dagan, 1990; Zhang et al., 1996; Zhang and Zhang, 1997].

[2] Although interesting for its general content this commentary addresses issues beyond the scope of our work, which focuses on assessing the interplay between the model of spatial variability and rate-limited sorption in shaping the evolution of the second moments of a reactive plume. Furthermore, since in applications the actual (real) distribution of hydraulic conductivity is unknown the idea of obtaining the actual plume moments is typically abandoned in favor of a stochastic description where the ensemble average of the spatial moments $S_{l l}=\left\langle s_{l l}\right\rangle$ represents the most probable estimate, while uncertainty can be characterized by its standard deviation $S D\left(s_{l l}\right)$, as shown by Suciu and Knabner [2009, Figure 1].

[3] An important result obtained by Fiori and Janković [2005], and confirmed later by Suciu et al. [2008] is that while $\left\langle s_{l l}\right\rangle$ converges to $X_{l l}$ as the source volume grows large, irrespective of its shape, the behavior of the standard deviation of $s_{l l}$ depends on the source's shape. In particular, convergence to zero of $S D\left(s_{l l}\right)$ is facilitated by large source dimensions in the direction $i \neq l$, and is hindered by large

\footnotetext{
${ }^{1}$ CIMA Research Foundation, International Centre on Environmental Monitoring, Savona, Italy.

${ }^{2}$ Department of Civil and Environmental Engineering, University of Trento, Trento, Italy.

${ }^{3}$ Department of Civil and Environmental Engineering, University of Illinois at Urbana-Champaign, Illinois, USA.
}

Copyright 2009 by the American Geophysical Union. 0043-1397/09/2009WR007815 dimension along $l$. For example, in a two-dimensional flow field $S D\left(s_{11}\right) \rightarrow 0$ for a planar source with $L_{1} \ll L_{2}$, where $L_{1}$ and $L_{2}$ are the source dimensions along the longitudinal and transverse directions, respectively. However, convergence to zero is hindered as $L_{1}$ increases, as evidenced in the Figure 1 of the commentary. Furthermore, in a threedimensional flow field ergodicity is obtained if at least one of the two source dimensions normal to $l$, the direction along which the moment is evaluated, is much larger than $L_{l}$. Therefore, although strictly speaking ergodicity in all directions is a rare event, as discussed in the commentary, one can identify several situations of practical importance in which the longitudinal spatial moment $s_{11}$ is very well approximated by the corresponding one particle displacement variance $X_{11}$. In all the other cases and directions, $X_{i i}, i=1, \ldots, m$, assumes the meaning of the most probable value of the unknown actual moment $s_{l l}$. For large sources $X_{l l}$ may be a suboptimal estimator of the corresponding spatial moment of the real plume, as remarked in the commentary, but at the same time it represents the best estimation one can obtain when the only information available on the spatial distribution of hydraulic properties are the parameters of the geostatistical model of spatial variability. In conclusion, we claim that the spatial moments and dispersivities we derived in our recent paper [Massabó et al., 2008] can be applied in a broader context than that indicated in the commentary by Suciu and Knabner [2009]. For example, as shown by Bellin and Tonina [2007], $X_{l l}$ can be used in the expressions of the first two concentration moments of Fiori and Dagan [2000] to obtain the pdf of the local solute concentration.

\section{References}

Bellin, A., and D. Tonina (2007), Probability density function of nonreactive solute concentration in heterogeneous porous formations, J. Contam. Hydrol., 94, 109-125.

Dagan, G. (1990), Transport in heterogeneous porous formations: Spatial moments, ergodicity and effective dispersion, Water Resour. Res., 26(6), $1281-1290$.

Fiori, A., and G. Dagan (2000), Concentration fluctuations in aquifer transport: A rigorous first-order solution and applications, J. Contam. Hydrol., $45(1-2), 139-163$.

Fiori, A., and A. Janković (2005), Can we determine the transverse macrodispersivity by using the method of moments?, Adv. Water Resour., 28, $589-599$.

Kitanidis, P. K. (1988), Prediction by the method of moments of transport in a heterogeneous formation, J. Hydrol., 102, 453-473. 
Massabó, M., A. Bellin, and A. J. Valocchi (2008), Spatial moments analysis of kinetically sorbing solutes in aquifer with bimodal permeability distribution, Water Resour. Res., 44, W09424, doi:10.1029 2007WR006539.

Suciu, N., and P. Knabner (2009), Comment on "Spatial moments analysis of kinetically sorbing solutes in aquifer with bimodal permeability distribution" by M. Massabó, A. Bellin, and A. J. Valocchi, Water Resour. Res., 45, W05601, doi:10.1029/2008WR007498.

Suciu, N., C. Vamoş, H. Vereecken, K. Sabelfeld, and P. Knabner (2008), Memory effects induced by dependence on initial conditions and ergodicity of transport in heterogeneous media, Water Resour. Res., 44, W08501, doi:10.1029/2007WR006740.

Zhang, Y. K., and D. Zhang (1997), Time-dependent dispersion of nonergodic plumes in two-dimensional heterogeneous aquifers, J. Hydrol. Eng., 2(2), 91-94.
Zhang, Y. K., D. Zhang, and J. Lin (1996), Nonergodic solute transport in three-dimensional heterogeneous isotropic aquifers, Water Resour. Res., 32(9), 2955-2963.

A. Bellin, Department of Civil and Environmental Engineering, University of Trento, Via Mesiano 77, Trento I-38050, Italy.

M. Massabó, CIMA Research Foundation, International Centre on Environmental Monitoring, University Campus, Via A. Magliotto 2, Savona I-17100, Italy. (marco.massabo@cimafoundation.org)

A. J. Valocchi, Department of Civil and Environmental Engineering, University of Illinois at Urbana-Champaign, 205 North Matthews Avenue, Urbana, IL 61801, USA. 